\title{
Energy clustering, Calibration and Particle Identification with the CMS Preshower detector
}

\section{Chiu-Ping Chang for the CMS collaboration}

National Central University, Jhongli City, Taiwan

E-mail: aaa19920824@gmail.com

\section{Shih-Yen Tseng for the CMS collaboration}

National Central University, Jhongli City, Taiwan

E-mail: a28945118@gmail.com

\begin{abstract}
The Preshower detector is located in front of the CMS endcap electromagnetic calorimeter. It is a sampling detector with two layers of lead absorber, each followed by $1.9 \mathrm{~mm}$ pitch silicon strip sensors. The 4288 DC-coupled sensors each have an active area of $61 \times 61 \mathrm{~mm}^{2}$, with a total surface area of around $16 \mathrm{~m}^{2}$, the largest ever built. The Preshower was installed to improve $\gamma / \pi^{0}$ discrimination. The Preshower energy clustering algorithms and calibration strategies are described. The algorithms have been improved to deal with bremsstrahlung and photon conversions due to the tracker material in front of the Preshower. Improved photon identification, for physics analyses such as Higgs decays to two photons, as well as improved electron identification in the endcap region of CMS are discussed.
\end{abstract}

The European Physical Society Conference on High Energy Physics -EPS-HEP2013

18-24 July 2013

Stockholm, Sweden 


\section{The CMS Preshower detector and dynamic $\phi$ road energy clustering}

The Preshower detector (ES) is located in front of the CMS endcap electromagnetic calorimeter (EE) [1]. It subtends a pseudo-rapidity range from $1.653<|\eta|<2.6$. The ES is a sampling detector with two layers of lead absorber $\left(2 X_{0} / 1 X_{0}\right)$, each followed by $1.9 \mathrm{~mm}$ pitch silicon strip sensors. The 4288 DC-coupled sensors each have an active area of $61 \times 61 \mathrm{~mm}^{2}$, with a total surface area of around $16 \mathrm{~m}^{2}$, the largest silicon detector built to date. The Preshower was installed to improve $\gamma / \pi^{0}$ discrimination. Clustering algorithms are used by CMS to sum together energy deposits in adjacent crystals belonging to the same electromagnetic shower. Basic clusters (BCs) are formed around the local maxima of energy deposits. These are merged to form a supercluster (SC), which is extended in $\phi$, to recover the radiated energy. In EE, the algorithm merges fixedsize $5 \times 5$ crystal BCs and associates each with the corresponding ES energy deposits. The new ES clustering algorithm uses an extended window in phi, to collect energy lost due to Bremsstrahlung, as follows:

1. The barycenters of all $\mathrm{BCs}$ belonging to a $\mathrm{SC}$ are extrapolated to the Preshower planes. The extrapolated point from the most energetic $\mathrm{BC}$ in the $\mathrm{SC}$ is chosen as the reference point.

2. The $\phi$ corresponding to all $\mathrm{BCs}$ in the SC are subtracted from the reference and the maximum $\Delta \phi$ in the clockwise and counterclockwise direction is used to define the clustering window along the $\phi$ direction. This window is finally extended by \pm 0.15 radians to take into account the fact that $\phi$ is computed at the center of each BC.

3. The energy deposits within the $\Delta \phi$ range defined in step 2 and with a pseudo-rapidity range, $\Delta \eta$, of \pm 0.15 with respect to the reference are summed to the total energy of the SC.

The improvement is presented in Figure 1, using Monte Carlo electron gun samples without pile-up. The two plots show the improvement in quadrature, $\sigma_{e f f}$. There is more improvement in the energy resolution at low pseudo-rapidity because there is more radiation in this region, due to the higher tracker material budget. The loss due to bremsstrahlung is defined as $\left|p_{\text {in }}-p_{\text {out }}\right| / p_{\text {in }}$ and $p_{\text {out }}$ are the momenta measured by the Tracker close to collision vertex and close to EE respectively.
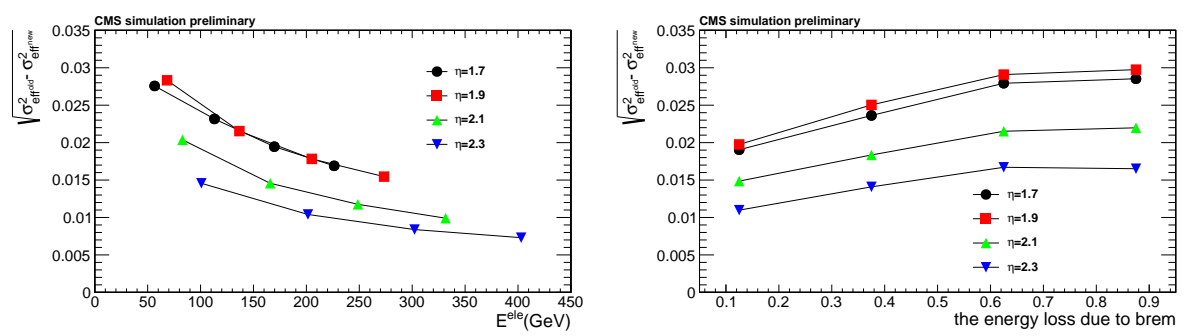

Figure 1: Left: The improvement of the new energy clustering for different $\eta$ values. Right: The improvement of the new energy clustering for different fractions of energy loss due to bremsstrahlung.

\section{Inter-calibration, dead-region calibration and photon identification using ES}

The energy of electromagnetic particles measured by EE can be expressed as:

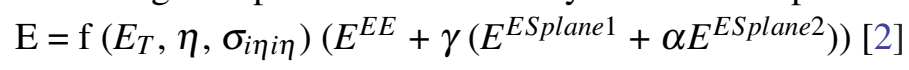


The $\gamma$ coefficient is extracted from a straight line fit as shown in Figure 2. It is measured to be $0.0188 \mathrm{GeV} / \mathrm{MIP}$, where MIP is the number of minimum ionising particles measured by ES for both ES planes working. A value of $\alpha$ of 0.7 was used thoughout these studies.


Figure 2: Left: Energy measured by EE crystals versus the energy deposits in ES (in units of the energy lost by minimum ionising particles (MIPs) in ES) by using MC unconverted $50 \mathrm{GeV}$ photons with $\eta$ between 1.7 and 2.3. Right: The combined EE + ES energy distribution for both planes working (green) and for both ES planes dead (black). In red, the resolution if only ES1 is working, but incorrectly using the gamma for the both planes working. In blue, the resolution obtained for ES1 working, but ES2 dead, using the correct $\gamma$ obtained from the dead channel calibration method.

However, there are some dead channels in the ES, mostly due to low-voltage supply problems. A special inter-calibration procedure for these regions is needed. Two different values of the $\gamma$ coefficient were estimated, depending on the ES plane which contributes to the energy measurement. Specifically, $\gamma$ is $0.043 \pm 0.0002$ for only ES plane 1 working and $0.029 \pm 0.0002$ for only ES plane 2 working. The two $\gamma$ coefficients are different because the first plane is after $2 X_{0}$, whereas the second plane is after $3 X_{0}$, of passive absorber. Figure 2 shows the energy distribution for different scenarios. By comparing the (Blue) and (Red) distributions, we find a 10.8\% improvement in energy resolution when the dedicated dead channel calibration is used (blue histogram).

The photon identification algorithm used in the CMS H $\rightarrow \gamma \gamma$ analysis benefit from the use of preshower information. In the endcap region, $\sigma_{R R}$, which is defined as the sum in quadrature of the second moment of the shower spread in the $\mathrm{x}$ and $\mathrm{y}$ planes of the preshower detector, is used as an input to the photon identification neural netwrk analysis for $\mathrm{H} \rightarrow \gamma \gamma$ analysis in CMS.

\section{Conclusion}

A new dynamic clustering algorith in ES has led to improvements in energy recovery due to bremstrahlung and conversions. The combined resolution of EE and ES has been improved by up to $10.8 \%$ with the introduction of new calibration coefficients to deal with the siuation where either of the preshower planes is not working.

\section{References}

[1] CMS collaboration, The CMS electromagnetic calorimeter project, Technical design report, CERN-LHCC-97-033.

[2] CMS Collaboration, Electromagnetic calorimeter calibration with 7 TeV data, CMS NOTE -2010/012 